\title{
ArcheoSciences
}

Revue d'archéométrie

\section{Études de taphonomie archéologique en milieu périglaciaire actuel : le Projet Gavarnie}

Archaeological Taphonomy in periglacial Environment: the Gavarnie Project

Arnaud Lenoble, Pascal Bertran, Cédric Beauval, Véronique Laroulandie, Stéphane Boulogne, Sandrine Costamagno, François LacrampeCuyaubère, Vincent Mourre, Olivier Onézime, Céline Thiébaut et JeanPierre Texier

\section{(2) OpenEdition}

Édition électronique

URL : https://journals.openedition.org/archeosciences/895

DOI : $10.4000 /$ archeosciences.895

ISBN : 978-2-7535-1597-0

ISSN : 2104-3728

Éditeur

Presses universitaires de Rennes

Édition imprimée

Date de publication : 31 décembre 2008

Pagination : 7-13

ISBN : 978-2-7535-0868-2

ISSN : 1960-1360

\section{Référence électronique}

Arnaud Lenoble, Pascal Bertran, Cédric Beauval, Véronique Laroulandie, Stéphane Boulogne, Sandrine Costamagno, François Lacrampe-Cuyaubère, Vincent Mourre, Olivier Onézime, Céline Thiébaut et Jean-Pierre Texier, «Études de taphonomie archéologique en milieu périglaciaire actuel : le Projet Gavarnie », ArcheoSciences [En ligne], 32 | 2008, mis en ligne le 31 décembre 2010, consulté le 01 février 2022. URL : http://journals.openedition.org/archeosciences/895 ; DOI : https://doi.org/ 10.4000/archeosciences.895 


\title{
Études de taphonomie archéologique en milieu périglaciaire actuel : le Projet Gavarnie
}

\author{
Archaeological Taphonomy in periglacial Environment: the Gavarnie Project
}

\author{
Arnaud Lenoble*, Pascal Bertran ${ }^{* *}, * * *$, Cédric Beauval ${ }^{* * * *}$, \\ Véronique Laroulandie**, Stéphane Boulogne***, Sandrine Costamagno*****, \\ François Lacrampe-Cuyaubère****, Vincent Mourre*****, Olivier OnÉZIme***, \\ Céline Thí́BAut****** et Jean-Pierre Texier**
}

\begin{abstract}
Résumé : Un programme d'archéologie expérimentale a été initié en milieu périglaciaire actuel. Le site sélectionné est localisé dans les Pyrénées centrales, sur le versant sud du Massif du Taillon, secteur de Gavarnie. Les expériences entreprises traitent des points suivants : (1) vitesse de déplacement des vestiges et évolution des structures anthropiques - amas de taille - par solifluxion et par reptation sur talus gravitaire; (2) fragmentation des ossements et altération des états de surface; (3) vitesse d'acquisition d'une orientation préférentielle des vestiges lithiques déplacés par solifluxion ou reptation. Après un an de fonctionnement, certains paramètres contrôlant la nature et la vitesse des modifications peuvent être identifiés. Pour les ossements, le caractère frais ou sec détermine les déplacements, tandis que le caractère brûlé / non brûlé apparaît prééminent par rapport à leur fragmentation. Dans le cas des vestiges lithiques déposés sur coulée de solifluxion, les déplacements diffèrent selon la disposition initiale des objets - en amas ou dispersés -. Ces premiers résultats permettent de réexaminer la validité des précédentes expériences de taphonomie archéologique en contexte périglaciaire.
\end{abstract}

\begin{abstract}
A$ set of long-term experiments have been initiated in an active periglacial environment. The experimental site is located on the south slope of the Taillon Mountain, near Gavarnie, Spain. Experiments address the following points: 1) the rate of displacement of archaeological particles and the disturbance of archaeological features on solifluction sheets and by creep on rockfall screes; 2) the fragmentation of bones and their surface alteration, with emphasis on cutmark disappearance; 3) the rate of linear fabric acquisition of knapped flint displaced by solifluction or creep. After one year, disturbances are observed. Considering bones, factors responsible for the changes noted are 1) green versus dry nature of items with regard to displacements 2) burnt versus unburnt status in respect of alteration. For lithic assemblage, we observe that initial artifact distribution - in regimented intervals or replicating knapping spot - controls the rate of displacement. This last point is crucial to the application of experimental data to archaeological sites. Previous experiments can be discussed in light of these preliminary results.
\end{abstract}

Mots clés : Processus de formation des sites, Archéologie expérimentale, Fragmentation, Taphonomie, Environnement périglaciaire, Paléolithique.

Keywords: Site formation processes, Experimental archaeology, Taphonomy, Periglacial environment, Palaeolithic.

* UMR 5198 du CNRS - Département de Préhistoire du Muséum National d'Histoire Naturelle - Musée de l'Homme, Palais de Chaillot, Place du Trocadéro, F-75116 Paris. (arnaud.lenoble@mnhn.fr)

** UMR 5199 du CNRS-PACEA - Université Bordeaux 1, Bât.B18, 33405 Talence cedex. (pascal.bertran@inrap.fr) (v.laroulandie@ipgq.u-bordeaux1. fr)(jp.texier@ipgq.u-bordeaux1.fr)

*** INRAP, Centre d'activités Les Echoppes, 156 Avenue Jean-Jaurès, F-33600 Pessac. (stephane.boulogne@inrap.fr) (olivier.onezime@inrap.fr)

****Archéosphère, Domaine du Haut-Carré, 351 cours de la Libération, F-33405 Talence Cedex. (c.beauval@archeosphere.com) (f.lacrampe@archeosphere.com)

***** UTAH, UMR 5608 du CNRS - Maison de la Recherche, Université Toulouse II - Le Mirail, 5 allée Antonio Machado, F-31058 Toulouse Cedex9. (costamag@univ-tlse2.fr) (vincent.mourre@wanadoo.fr)

****** ESEP, UMR 6636 du CNRS - université Aix-Marseille 5, rue du Château de l'Horloge, BP 647, F-13094 Aix-en-Provence Cedex 2. (celine. thiebaut@wanadoo.fr) 


\section{INTRODUCTION}

De nombreux sites archéologiques se sont formés en milieu périglaciaire. Les modifications qui précèdent ou qui accompagnent l'enfouissement des vestiges dans ce type de milieu restent cependant difficiles à apprécier. Cela tient au nombre très réduit de travaux expérimentaux consacrés à ce sujet. Des expériences en laboratoire ont traité la fragmentation des ossements (Guadelli et Ozouf, 1994) ou la cryoexpulsion des objets enfouis dans un sédiment soumis à des cycles de gel/dégel (Johnson et Hansen, 1974). Mais les travaux d'archéologie expérimentale se sont surtout intéressés aux phénomènes de redistribution des vestiges. Ainsi, Bowers et collaborateurs (1983) ont quantifié les déplacements sur sols plats liés à la formation d'aiguilles de glace tandis que le programme TRANSIT (Transfert du Référentiel Alpin et Nival aux SITes archéologiques) a abordé les déplacements par solifluxion (Texier et al., 1998).

Ces résultats expérimentaux ne sont toutefois pas directement applicables aux sites archéologiques, pour deux raisons :

1. la durée très courte des expériences au regard de celle mise en jeu dans la formation des sites archéologiques. Face à cette difficulté, les auteurs ont eu recours aux simulations informatiques. Bowers et al. (1983) montrent ainsi que par reptation sur sol plat, un amas de débitage se déforme progressivement par accroissement de sa surface couverte et affaiblissement corrélatif de la concentration en vestiges. Dans le cas des modifications par solifluxion où les déplacements sont préférentiellement orientés, l'ensemble des objets se déplace dans la pente tandis que la structure prend, dans un premier temps, une forme allongée avec une concentration plus importante des vestiges dans la partie amont, avant que la dispersion des pièces ne soit complète (Lenoble et al., 2003). Toutefois, les étapes de dégradation prédites par ces modèles ne sont pas directement comparables aux sites archéologiques retrouvés en contexte de solifluxion. Ceci tiendrait à la disposition initiale - en ligne - des objets au cours des expériences, qui s'écarte de celle constatée dans les sites archéologiques peu perturbés. Une telle disposition conduit à ignorer l'interaction entre les structures archéologiques (amas, fosse, etc.) et la dynamique sédimentaire (Lenoble et al., 2003).

2. la reconnaissance, au cours de l'étude des sites archéologiques, de l'intensité des modifications implique de disposer de critères diagnostiques spécifiques à chacune des étapes de dégradation. De ce point de vue, très peu de choses ont été faites. Ainsi, les déplacements par solifluxion conduisent à une orientation préférentielle des objets allongés selon la pente (Bertran et al., 1997), mais la vitesse d'acquisition de cette fabrique n'est pas connue. Aussi l'observation d'une orientation préférentielle des pièces ne permet pas, à ce jour, d'en déduire l'ampleur de la redistribution corrélative des vestiges. De même, dans le cas des vestiges osseux, la vitesse et les stades d'altération des surfaces corticales sont peu documentés et, pour cette raison, il est difficile de mettre en parallèle le degré d'altération des surfaces osseuses avec l'importance de la déformation des structures archéologiques.

Finalement, ces expériences attestent des modifications que peuvent subir les sites en milieu périglaciaire et la simulation informatique, basée sur l'extrapolation des mesures expérimentales, permet de proposer des modèles de redistribution des vestiges. Mais, faute de critères diagnostiques, les étapes de dégradation prédites par ces modèles restent difficiles à reconnaître au cours des études de sites.

Ce constat nous a conduit à entreprendre un nouveau programme d'archéologie expérimentale. Son objectif est de compléter les précédents travaux réalisés en milieu actif. Pour cela, une attention particulière est apportée, d'une part, aux différents critères qui peuvent être pris en compte au cours de l'étude des sites archéologiques (états de surface, fabrique, tri granulométrique, etc.) et, d'autre part, à l'influence des variables spécifiques aux configurations archéologiques (état de fraîcheur des ossements, disposition initiale des vestiges). En outre, la localisation des expériences - position du site en entrée de grotte ou sur versant - est également prise en compte. Dans ce cadre de cette note sont présentés le site expérimental retenu, le protocole mis en œuvre et les observations et mesures réalisées un an après que l'expérience ait été initiée. Ces premiers résultats permettent de cerner les principales modalités de dégradation des ensembles expérimentaux, d'en discuter les facteurs de contrôle et, sur cette base, de situer les apports du projet Gavarnie vis-à-vis des précédentes expériences de taphonomie archéologique en milieu périglaciaire.

\section{LE SITE EXPÉRIMENTAL}

Le site expérimental est localisé sur le versant sud du massif du Taillon, dans le secteur de Gavarnie (Pyrénées centrales) (figure 1). Ce secteur a été retenu car il se place en milieu périglaciaire d'altitude (>2500 m) et présente une lithologie dominée par les calcaires gréseux et lithographiques avec un karst bien développé.

Un secteur de replats structuraux offrant des configurations de pentes variables a été retenu pour accueillir les différentes cellules, à une altitude de $2800 \mathrm{~m}$ (figure 2). Des pavages caillouteux couvrent le sol. La végétation est réduite à quelques taches d'herbe. Les affleurements rocheux sont soumis à une gélifraction intense. Les débris forment des talus d'éboulis gravitaires aux pieds des abrupts et sont redistribués sur les versants par les processus périglaciaires. Les coulées de solifluxion sont nombreuses. Elles s'organisent en coulées à front pierreux sur pente faible à moyenne et en terrassettes sur forte pente. 


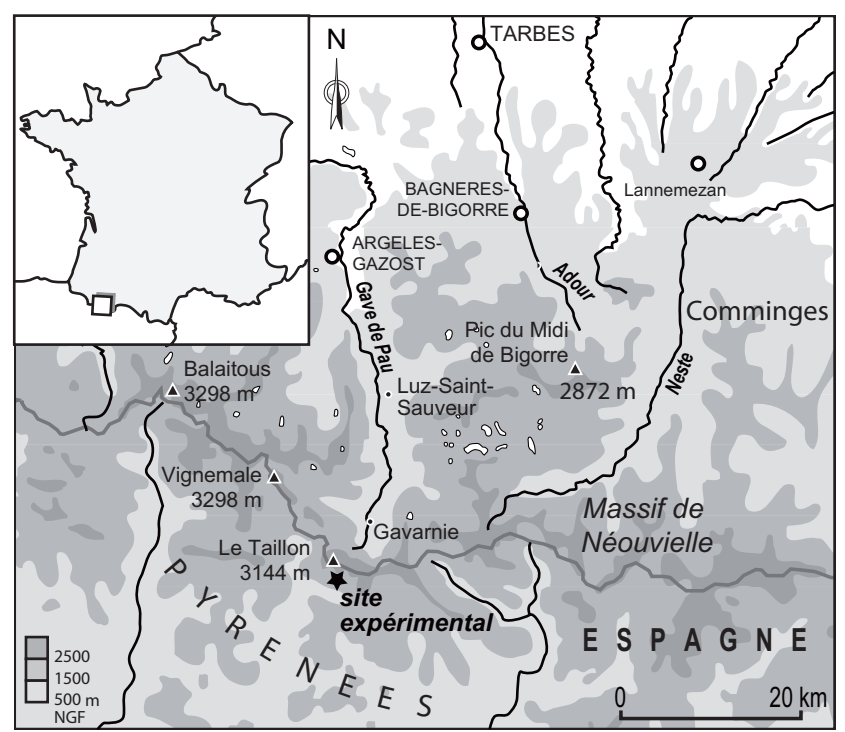

Figure 1 : Localisation du site expérimental.

Figure 1: Experimental site location.

L'isotherme et la pluviométrie sont évaluées à partir des stations météorologiques proches de Gèdre et de Gavarnie. La comparaison des enregistrements entre ces deux stations permet d'établir un gradient thermique de $0,52^{\circ} \mathrm{C}$. Cette valeur est comparable à celle de $0,5^{\circ} \mathrm{C}$ proposée par Julian Andrés et Chueca Cia (1998) pour le versant méridional des Pyrénées centrales et au gradient de $0,56{ }^{\circ} \mathrm{C} / 100$ obtenu par Barrio et al. (1990) pour l'ensemble des Pyrénées centrales. Le gradient d'altitude de pluviométrie, calculé de la même façon, est de $25 \mathrm{~mm}$ par $100 \mathrm{~m}$. Sur cette base, la température moyenne annuelle du site expérimental est comprise entre 0 et $-1{ }^{\circ} \mathrm{C}$ et les précipitations y sont, au minimum, de $1500 \mathrm{~mm} / \mathrm{an}$.

Un enregistrement précis des températures est réalisé à partir d'un enregistreur relié à huit sondes thermiques. Celles-ci sont disposées de manière à obtenir deux profils de températures, l'un dans la grotte sélectionnée pour accueillir l'un des locus expérimentaux ( $c f$. infra) et l'autre à l'extérieur de la cavité, sur le talus orienté vers l'Est. Les sondes sont enfouies en subsurface, à 10 et à $30 \mathrm{~cm}$ de profondeur et, pour chaque profil, une des sondes est placée dans la paroi près de sa surface. Les enregistrements recueillis indiquent que les alternances gel/dégel ont lieu essentiellement au début de l'automne et se limitent à un peu plus d'une dizaine par an. Ce nombre limité de cycles est lié à l'importante couverture neigeuse présente du début de l'automne jusqu'à la fin du printemps. Cette couverture limite la pénétration du gel dans le sol. Ceci est d'autant plus vrai à l'entrée de la grotte que cette dernière s'ouvre sur une petite dépression jouant le rôle de piège à neige. Une variabilité annuelle importante est cependant attendue, en réponse à la plus ou moins grande

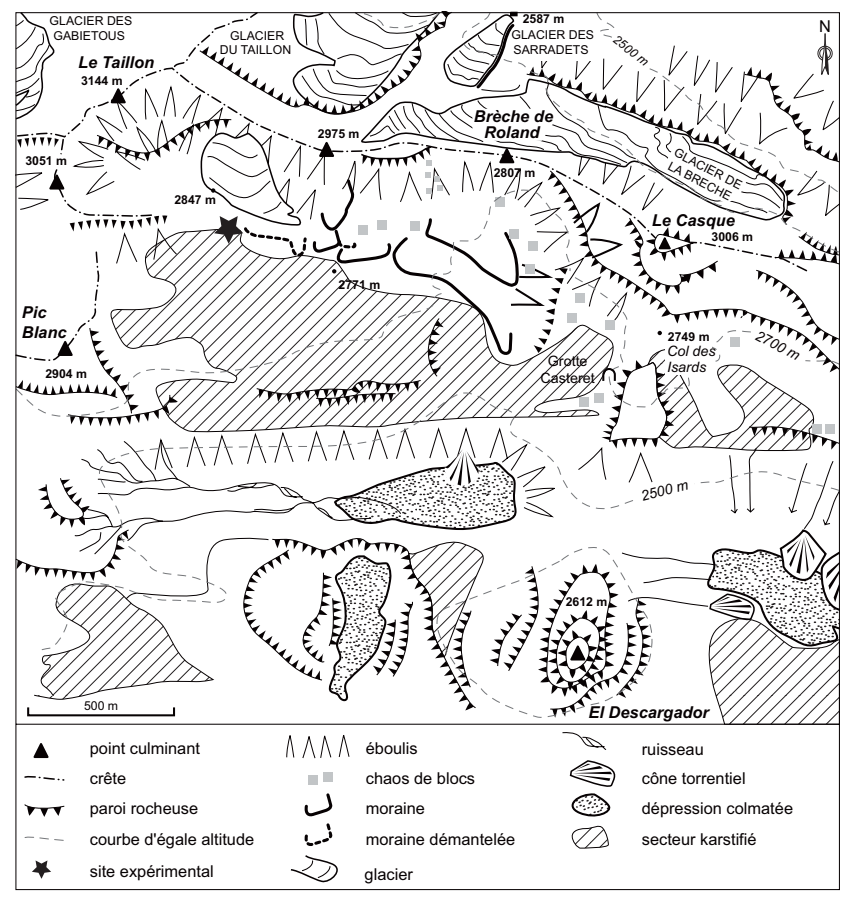

Figure 2 : Carte géomorphologique du versant sud de la brèche de Rolland. Cette carte utilise des informations publiées par GarciaRuiz et Marti Bono (2001) ainsi que par Majesté-Menjoulas et al. (1999).

Figure 2: Geomorphological map, southern slope of the Taillon - brèche de Rolland area.

précocité de la fonte du manteau neigeux, cette dernière autorisant la pénétration des cycles printaniers de gel dans le sol.

\section{LES EXPÉRIENCES}

Trois expériences ont été initiées à l'automne 2003. Quatre locus ont été retenus à cette fin : trois sur des versants, pour des valeurs de pentes variées et un sur un talus constitué de fragments rocheux en entrée de grotte (tableau 1). Sur les versants, les vestiges on été disposés préférentiellement sur le dos des coulées où le pavage superficiel est formé de petits cailloux et de granules. La microtopographie qui en résulte, très faible, n'est pas de nature à interférer avec le déplacement des pièces. La disposition des vestiges répond à trois objectifs différents (figure 3 et tableau 1) :

1) Expérience " amas »: Cette expérience a pour objet de tester l'influence de la disposition des vestiges en amas sur les déplacements et de documenter le tri granulométrique qui accompagne la dispersion des objets. Pour cela, deux amas ont été reconstitués, l'un en entrée de grotte et l'autre sur la coulée de solifluxion du locus 1 . 


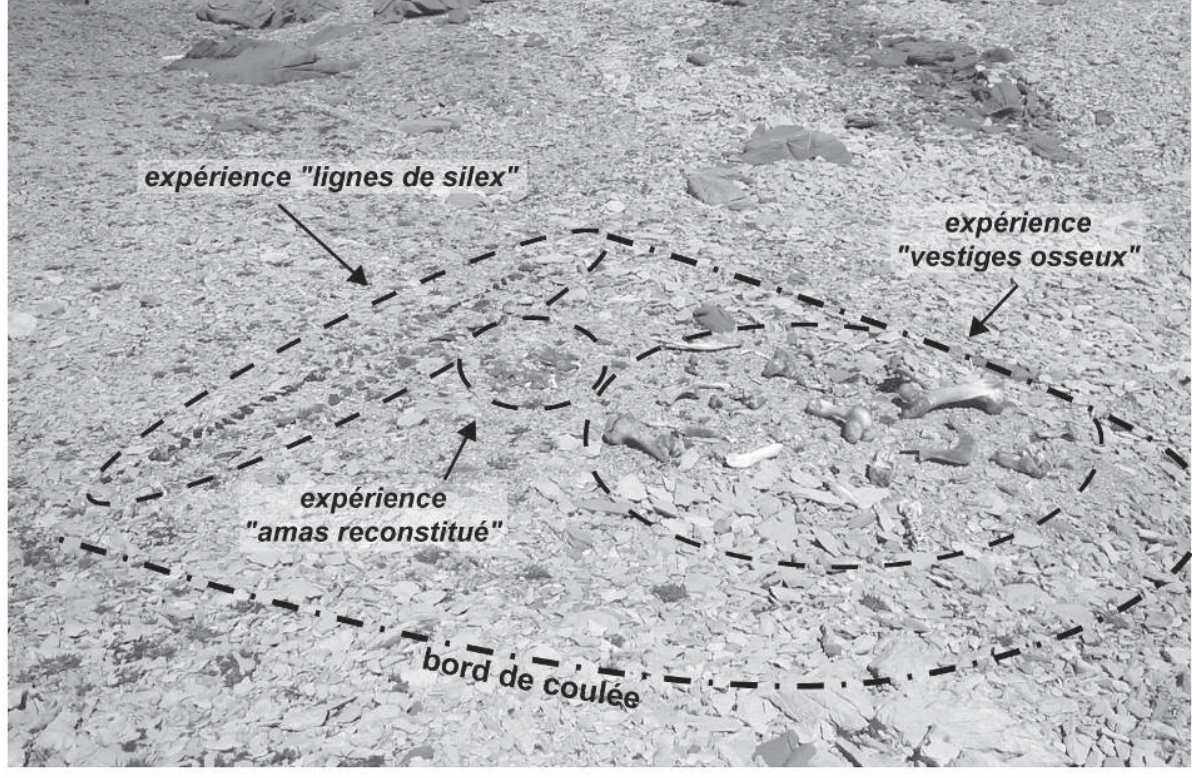

Figure 3: Vue du locus expérimental 1 lors de sa mise en place. La coulée à front pierreux sur laquelle les objets sont placés est large de trois mètres.

Figure 3: View of cell 1 at the experience initiation. The stone-banked sheet on which artifacts are located is about three metres wide.
Pour chacun des deux amas, les éclats utilisés sont issus de la taille d'un même bloc de silex. Les fragments plus petits que $2 \mathrm{~cm}$ sont peints d'une couleur propre à chaque classe dimensionnelle retenue $(2-4 \mathrm{~mm} ; 4-5 \mathrm{~mm} ; 5-10 \mathrm{~mm}$ et 10 $20 \mathrm{~mm}$ ). Les objets plus gros, quant-à eux, sont mesurés et numérotés. L'ensemble est replacé sur le site expérimental conformément au modèle de distribution d'un amas de taille (Boëda et Pellegrin, 1985). Au cours du positionnement, la localisation de chaque vestige numéroté est enregistrée tandis que les fragments plus petits sont décomptés selon une grille de maille $10 \mathrm{~cm}$.

Un troisième amas est issu de la taille d'un bloc de silex à même le site, sur la coulée de solifluxion du locus 2 . Il sert de base de comparaison pour vérifier que le protocole de reconstitution des amas n'a pas d'influence sur les déformations observées. L'enregistrement des éclats ne peut toutefois être aussi poussé car, du fait de l'enchevêtrement et des nombreux recouvrements de pièces, un tel enregistrement provoquerait une désorganisation de la structure. Aussi cet amas fait l'objet d'un relevé photographique tandis qu'une dizaine d'éclats présents en surface est numérotée et coordonnée.
Les amas sont photographiés et les objets numérotés sont coordonnés à chaque nouvelle visite du site. Leur démontage clôturera l'expérience.

2) Expérience "lignes de silex » : cette expérience a un double objectif : a) obtenir des mesures de déplacement de pièces isolées, et b) caractériser la durée d'acquisition d'une orientation préférentielle de pièces disposées isolément. À cette fin, des lames de silex sont disposées en lignes. Ces lignes sont placées perpendiculairement à la pente. La position originelle du grand axe des lames - parallèle, perpendiculaire ou oblique par rapport à la direction de pente - est choisie de façon à ce que les différentes configurations soient également représentées. Le déplacement, l'orientation et l'inclinaison des pièces sont mesurées chaque année.

3) Expérience "vestiges osseux ": l'objectif est d'enregistrer a) l'altération des surfaces corticales et l'oblitération des traces qui s'y trouvent, b) la fragmentation des restes au cours du temps, et c) leurs déplacements.

Les pièces osseuses sont disposées aléatoirement. Elles appartiennent à plusieurs taxons (boviné, ovicapriné, oiseaux), sont issues de segments anatomiques distincts

\begin{tabular}{|l|c|c|c|c|}
\hline \multicolumn{1}{|c|}{ Locus } & Pente $\left(^{\circ}\right)$ & Expérience « amas » & Expérience « lignes » & Expérience "vestiges osseux » \\
\hline Locus 1 & 5 & Amas reconstitué & Lames de silex disposées en lignes & Disposition aléatoire d'ossements \\
\hline Locus 2 & 10,5 & Amas taillé sur place & Lames de silex disposées en lignes & - \\
\hline Locus 3 & 21 & - & - & Disposition aléatoire d'ossements \\
\hline $\begin{array}{l}\text { Locus } 4 \\
\text { grotte) }\end{array}$ & 24,5 & Amas reconstitué & Lames de silex disposées en lignes & Disposition aléatoire d'ossements \\
\hline
\end{tabular}

Tableau 1 : composition des différents locus expérimentaux mis en place en 2003.

Table 1: experimental cells composition. 
et représentent des classes d'âges variées. La fraîcheur des ossements est variable et quatre catégories sont distinguées : frais, sec, brûlé et fossile. Les vestiges portent les stigmates d'agents taphonomiques divers (carnivore, homme, rongeur, végétaux). Chaque élément a été décrit, dessiné et photographié préalablement à sa mise en place.

\section{RÉsultats}

\section{Déplacements}

Après un an de fonctionnement, tous les objets se sont déplacés, au minimum d'un demi-centimètre et parfois de plusieurs dizaines de mètres (figure 4). Les paramètres à
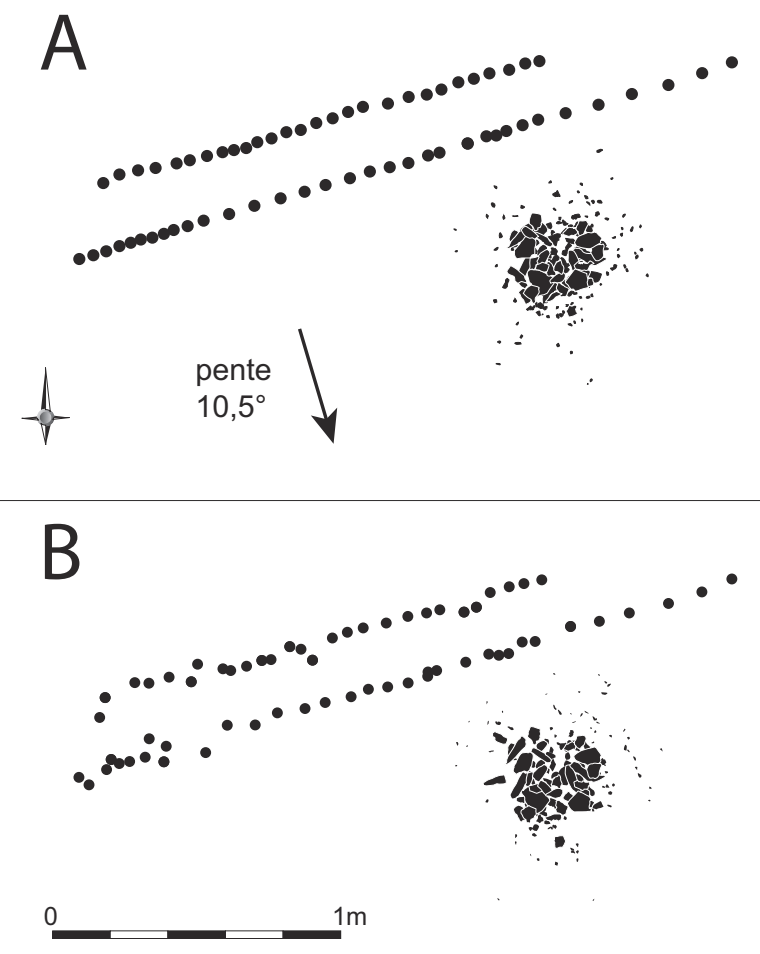

Figure 4 : Plan des vestiges composant l'amas reconstitué et les lignes de silex de la cellule 1 en septembre 2003 (A) et en septembre 2004 (B). Sur cette figure, Seuls les vestiges plus grands que $5 \mathrm{~mm}$ sont figurés. Les éléments composants les lignes de silex sont représentés par des points. L'évolution montre une dispersion des objets, les contours de l'amas devenant plus diffus, ainsi qu'une disparition des plus petits vestiges qui s'infiltrent dans le pavage caillouteux. Un mouvement d'ensemble vers l'aval est perceptible.

Figure 4: locus 1 artifact distribution for September 2003 (A) and September 2004 (B). For the knapping spot, only artifacts larger than $5 \mathrm{~mm}$ are plotted. Piece compounding fint lines are shown by a point. The modification consists of a scattering and a subtraction of smaller flakes trapped in the stone surface pavement. An overall downslope movement can be observed. prendre en compte sont les mécanismes de déplacement, la disposition initiale des objets et la nature des vestiges, lithique ou osseux.

1) Les mécanismes de déplacement. Les objets disposés isolément se sont déplacés de façon comparable sur le talus d'entrée de grotte et sur les coulées. Sur les versants, le déplacement des pièces est imputable à la solifluxion puisque cette dernière contrôle l'évolution des versants, comme l'indique la présence de terrassettes et de coulées. Dans la cavité, le déplacement peut être imputé à une reptation de l'éboulis causée par le poids de la neige, aux égouttements et à la chute de cailloux, etc. (Bertran et al. 2004), mais aussi à l'instabilité des objets récemment positionnés. Si cette dernière hypothèse est la bonne, les déplacements devraient rapidement diminuer d'importance (Caine 1981). Aussi, la poursuite des mesures permettra-t-elle de trancher entre ces différentes hypothèses.

2) La disposition initiale des objets sur les coulées de solifluxion (figure 5). Les objets disposés individuellement (expérience "lignes de silex ») se déplacent conformément aux mouvements du sol induits par les cycles de gel/dégel : le mode des déplacements est bien marqué, entre 1,5 et $2,5 \mathrm{~cm}$, l'écart-type est faible et l'orientation des déplacements est conforme à la pente. En revanche, dans le cas des objets regroupés (expérience " amas »), les déplacements sont en moyenne plus faibles (mode $1 \mathrm{~cm}$ ) et les plus grands déplacements, en l'occurrence supérieur à $3 \mathrm{~cm}$, ne concernent que les objets placés en périphérie des concentrations. Par ailleurs, la direction moyenne des déplacements peut

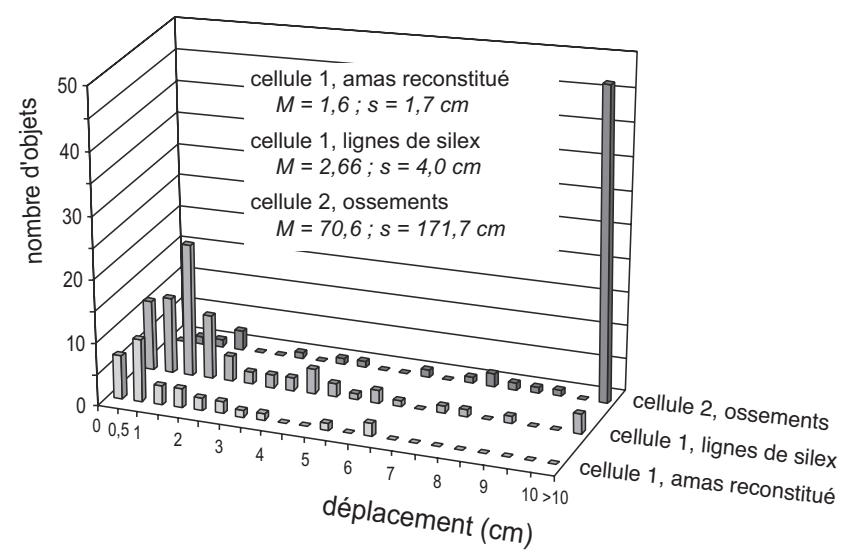

Figure 5 : Distance des déplacements sur coulée de solifluxion. Les valeurs suivent des distributions log-normales. Aussi, pour chaque série, la moyenne (M) et l'écart-type (s) géométriques sont indiqués.

Figure 5: Displacements of artifacts located on solifluction sheet. Data set fit to log-normal distribution. Geometric mean value $(M)$ and geometric standard deviation (s) are then indicated. 
s'écarter sensiblement de la pente. Nous proposons d'appeler « effet amas " cette influence du mode de disposition des pièces. Cet effet peut s'expliquer par l'imbrication des objets et la microtopographie induite par les nombreuses pièces formant la concentration, ces deux facteurs limitant le déplacement de chaque vestige. Mais elle peut également s'expliquer par la texture ouverte de l'amas, c'est-à-dire par l'absence de sédiment fin entre les objets de la concentration, qui ne permet pas la formation de glace de ségrégation.

3) La nature des vestiges : lithiques ou osseux. Les grandes distances de déplacement concernent les vestiges osseux (figure 5). Elles sont imputables aux agents biologiques. En effet, sur les versants, le déplacement moyen est de $72 \mathrm{~cm}$ pour la cellule contenant des os frais (locus 1) alors qu'il est comparable à celui des vestiges lithiques pour la cellule n'en contenant pas (locus 3). Par ailleurs, environ un tiers des ossements déposés sur la coulée de solifluxion (locus 1), essentiellement des os frais, n'ont pas été retrouvés. Parmi eux, une mandibule de boviné, sèche, et un silex ont été retrouvés à une centaine de mètres du locus 1 où ils avaient été placés. Cette cellule est celle dans laquelle les charognards (vautour, gypaète, corvidé ou renard) ont prélevé les os frais. Nous supposons que ces deux pièces ont été emportées comme les autres ossements, mais rapidement abandonnées du fait de l'absence d'intérêt nutritif.

\section{Altération des vestiges osseux}

Des dégradations sont d'ores et déjà perceptibles au cours de cette première année. La nature des vestiges (frais, sec, brûlé ou fossile) a fortement influencé leur altération. Les os frais ont intéressé les charognards qui ont emporté certaines pièces et laissé des traces de manducation sur d'autres. Les os brûlés, fragiles, se sont fortement fragmentés. Des os fossiles, dont la structure est modifiée par disparition de la fraction organique, se sont fissurés et présentent parfois des plans de délitement (figure 6). Finalement, ce sont les os secs qui ont le moins subis de modifications à la suite de cette première année d'expérience. Ils ne sont, en effet, ni rongés, ni fissurés.

\section{Discussion}

Les résultats acquis cette première année doivent être confirmés par un temps d'expérience plus important. Toutefois, plusieurs résultats méritent d'être soulignés :

1. la confirmation d'un " effet amas " conduit à relativiser la portée des expériences antérieures. Ainsi, Bowers et al. (1983) quantifient les déplacements d'objets archéologi-

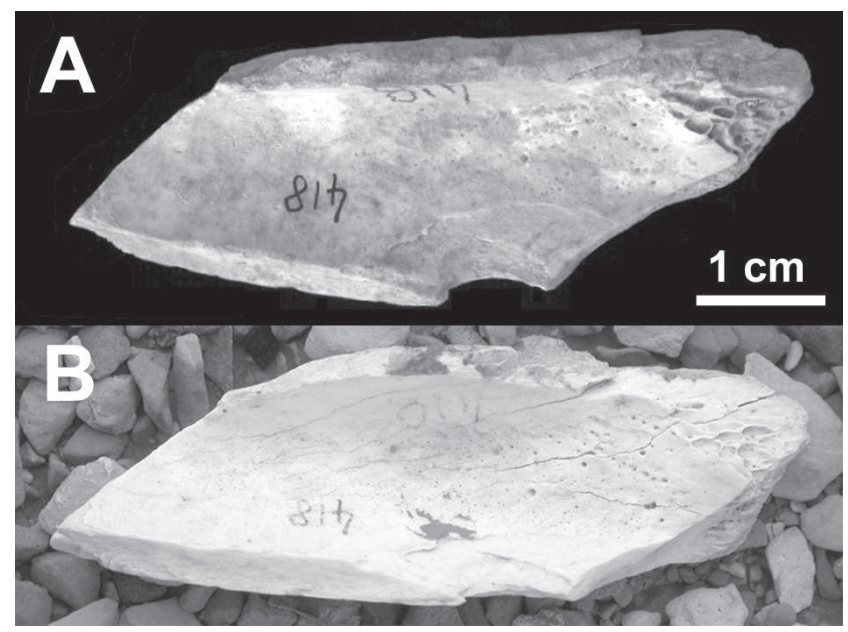

Figure 6: Os fossile photographié avant la mise en place (A) et après 1 an d'exposition (B). Noter le développement de fissures longitudinales.

Figure 6: Close-up view of a fossil bone before experience initiation (A) and after one year of exposure (B). Note the appearance of longitudinal fissures.

ques par formation répétée d'aiguilles de glace à partir de la mesure du déplacement d'une série d'objets isolés. Ces auteurs extrapolent les mesures ainsi acquises pour simuler la déformation d'un amas de taille sur des durées de plusieurs millénaires. Il en ressort une diffusion rapide et importante de la concentration originelle. La mise en évidence d'un " effet amas" pose cependant question sur la validité d'une telle extrapolation. L'influence importante d'une interaction entre les pièces peut conduire à des déplacements moindres mais, également, à la formation de concentrations secondaires par immobilisation de groupes d'objets comme le prédit Anhert (1994) dans ses simulations informatiques sur la genèse de sols structurés. De nouvelles simulations sont donc nécessaires pour valider ou préciser le modèle proposé par Bowers et al. (1993), tant en terme d'évolution des structures (diffusion homogène et progressive par dispersion?) que de vitesse de modification. Ces simulations doivent se baser sur des données expérimentales qui restituent l'influence de l'interaction entre les vestiges. L'acquisition de telles données est en cours dans le cadre du projet Gavarnie.

2. Dans le cas des ossements, l'état de fraîcheur des pièces influence fortement la préservation des vestiges. De fait, les déplacements d'os secs par solifluxion qui ont été observés au cours de l'expérience TRANSIT (Texier et al., 1998), bien que spécifiques au milieu périglaciaire, n’illustrent que partiellement les dégradations auxquelles sont exposés les 
vestiges osseux dans ce type de milieu. En particulier, dans un laps de temps très court et avant toute fossilisation, les os frais sont fortement dispersés par l'action de la faune. Dans ce même temps très court et avec seulement quelques dizaines de cycles de gel/dégel par an, les os brûlés sont très fragmentés, à l'inverse des os frais. Une préservation préférentielle de ces derniers pourrait ainsi caractériser les ensembles osseux ayant été exposés à l'action d'un froid, même modéré.

\section{Remerciements}

Le programme d'archéologie expérimentale de Gavarnie est financé par l'Action Collective de Recherche «le Paléolithique moyen en Aquitaine Septentrionale ». Nous remercions également les responsables du Parque Nacional d'Ordesa y Monte Perdido qui nous ont aimablement transmis les valeurs météorologiques de la station de Goriz.

\section{Bibliographie}

Ahnert (F.), 1994. Modelling the development of non-periglacial sorted nets. Catena, 23, p. 43-63.

Barrio (G. Del), Creux (J.) et Puigdefabregas (J.), 1990. Thermal seasonality of the high mountain belts of the Pyrenees. Moutain Research and Development, 10 (3), p. 227-233.

Bertran (P.), Hétu (B.), Texier (J.-P.) et Van Steijn (H.), 1997. Fabric characteristics of subaerial slope deposits. Sedimentology, 44, p. 1-16.

Bertran (P.) Texier (J.-P.) et Francou (B.), 2004. Éboulisation - éboulement, in Bertran (P.) (dir.), Dépôts de pente continentaux : dynamique et faciès, Quaternaire, suppl. 1, p. 29-43.

Bö̈da (E.) et Pellegrin (J.), 1985. Approche expérimentale des amas de Marsangy, in Archéologie expérimentale. Les amas de la zone N19 du gisement magdalénien de Marsangy: appro- che méthodologique par l'expérimentation, Association pour la promotion de l'archéologie en Bourgogne, Archéodrome, p. 19-36.

Bowers (P. M.), Bonnichsen (R.) et Hoch (D. M.), 1983. Flake dispersal experiments: non-cultural transformation of the archaeological record, Antiquity, 48 (3), p. 553-572.

CaIne (N.), 1981. A source of biais in rates of surface soil movement as estimated from marked particles, Earth Surface Processes and Landforms, 6, p. 69-75.

Garcia-Ruiz (J. M.) et Marti-Bono (C. E.), 2001. Mapa geomorfologico del Parque Nacional de Ordessa y Monte Perdido. Organismo Autonomo de Parques Nacionales, Madrid.

Guadelli (J.-L.) et Ozouf (J.-C.), 1994. Études expérimentales de l'action du gel sur les restes fauniques : premiers résultats, Artefact, 9, p. 47-56.

JoHnson (D. L.) et HANSEN (K. L.), 1974. The effects of frostheaving on objects in soils, Plains Anthropologist, 19 (64) p. 81-98.

Julian Andres (A.) et Chueca Cia (J.), 1998. Le petit âge glaciaire dans les Pyrénées Centrales mériodionales : estimation des paléotempératures à partir d'inférences géomorphologiques, Sud-Ouest Européen, 3, p. 79-88.

Lenoble (A.), Bertran (P.), Lacrampe (F.), Bourguignon (L.) et Detrain (L.), 2003. Impact de la solifluxion sur les niveaux archéologiques : simulation à partir d'une expérience en milieu actif et application à des sites paléolithiques aquitains. Paléo, 15 , p. 105-122.

Majesté-Menjoulas (C.), Debon (F.) et Barrère (P.), 1999. Carte géologique de la France au 1/50 000', feuille de Gavarnie. BRGM, Orléans.

Texier (J.-P.), Bertran (P.), Coutard (J.-C.), Francou (B.), Gabert (P.), Guadelli (J.-L.), Ozouf (J.-C.), Plisson (H.), Raynal (J.-P.) et Vivent (D.), 1998. TRANSIT, an experimental archaeological program in periglacial environment: problematic, methodology, first results, Geoarchaeology, 13 (5), p. 433-473. 